\title{
GSK3 $\beta$ Regulates Myelin-Dependent Axon Outgrowth Inhibition through CRMP4
}

\author{
Yazan Z. Alabed, ${ }^{1 \star}$ Madeline Pool, ${ }^{1 \star}$ Stephan Ong Tone, ${ }^{1}$ Calum Sutherland, ${ }^{2}$ and Alyson E. Fournier ${ }^{1}$ \\ ${ }^{1}$ Department of Neurology and Neurosurgery, Montreal Neurological Institute, Montreal, Quebec H3A 2B4, Canada, and ${ }^{2}$ Neurosciences Institute, Division \\ of Pathology and Neuroscience, University of Dundee, Ninewells Hospital and Medical School, Dundee DD1 9SY, Scotland
}

Myelin-associated inhibitors (MAIs) contribute to failed regeneration in the CNS. The intracellular signaling pathways through which MAIs block axonal repair remain largely unknown. Here, we report that the kinase GSK3 $\beta$ is directly phosphorylated and inactivated by MAIs, consequently regulating protein-protein interactions that are critical for myelin-dependent inhibition. Inhibition of GSK3 $\beta$ mimics the neurite outgrowth inhibitory effect of myelin. The inhibitory effects of GSK3 $\beta$ inhibitors and myelin are not additive indicating that GSK3 $\beta$ is a major effector of MAIs. Consistent with this, overexpression of GSK3 $\beta$ attenuates myelin inhibition. MAI-dependent phosphorylation and inactivation of GSK $3 \beta$ regulate phosphorylation of CRMP4, a cytosolic regulator of myelin inhibition, and its ability to complex with RhoA. Introduction of a CRMP4 antagonist attenuates the neurite outgrowth inhibitory properties of GSK3 $\beta$ inhibitors. We describe the first example of GSK $3 \beta$ inactivation in response to inhibitory ligands and link the neurite outgrowth inhibitory effects of GSK $3 \beta$ inhibition directly to CRMP4. These findings raise the possibility that GSK3 $\beta$ inhibition will not effectively promote long-distance CNS regeneration following trauma such as spinal cord injury.

\section{Introduction}

Inhibitory molecules at CNS lesion sites including myelinassociated inhibitors (MAIs) and chondroitin sulfate proteoglycans (CSPGs) activate RhoA in injured neurons to mediate neurite outgrowth inhibition (Liu et al., 2006). In a screen to identify proteins that functionally interact with RhoA in the context of neurite outgrowth inhibition, we previously identified the cytosolic phosphoprotein CRMP4 (Collapsin Response Mediator Protein 4) as a protein that functionally interacts with RhoA to mediate neurite outgrowth inhibition (Alabed et al., 2007). The CRMP family consists of five family members (CRMP1-5) in vertebrates (Goshima et al., 1995; Minturn et al., 1995; Byk et al., 1996; Gaetano et al., 1997; Inatome et al., 2000) that regulate aspects of axon pathfinding and neurite outgrowth (Hedgecock et al., 1985; Siddiqui and Culotti, 1991; Goshima et al., 1995; Minturn et al., 1995; Quinn et al., 1999, 2003; Yoshimura et al., 2005). Each CRMP allele produces two transcripts which differ in their $\mathrm{N}$ terminus, yielding long (L-CRMP) and short (S-CRMP) isoforms, which have alternatively been referred to as " $a$ " and " $b$ " isoforms (Quinn et al., 2003; Yuasa-Kawada et al., 2003; Alabed et al., 2007; Pan et al., 2010).

\footnotetext{
Received Dec. 11, 2009; revised March 9, 2010; accepted March 12, 2010.

This study was supported by a grant to A.E.F. from the Canadian Institutes of Health Research (CIHR). A.E.F. is a Tier 2 Canada Research Chair. Y.Z.A. and S.O.T. are supported by fellowships from CIHR, and M.P. is supported by a fellowship from the Multiple Sclerosis Society of Canada. We thank Dr. Keith Burridge for RhoA constructs, Dr. Dennis Stacey for GSK3 $\beta$ constructs, Isabel Rambaldi for technical assistance, Brigitte Ritter for assistance with lentivirus, Daniel Lee for assistance with the generation of the phospho-specific L-CRMP4 antibody, Dr. Craig Mandato for helpful discussions, and Drs. Phil Barker and Wayne Sossin for helpful comments on this manuscript.

*Y.Z.A. and M.P. contributed equally to this work.

Correspondence should be addressed to Alyson E. Fournier, Montreal Neurological Institute, BT-109, 3801 Rue University, Montreal, QC H3A 2B4, Canada. E-mail: alyson.fournier@mcgill.ca.

D01:10.1523/JNEUROSCI.6154-09.2010

Copyright $\odot 2010$ the authors $\quad 0270-6474 / 10 / 305635-09 \$ 15.00 / 0$
}

Treatment of neurons with the MAI Nogo specifically enhances the association between RhoA and L-CRMP4 (Alabed et al., 2007); however, the mechanism(s) regulating the formation of this complex is unknown and will add insight into the signaling mechanisms mediating neurite outgrowth inhibition. We find that the L-CRMP4-RhoA protein interaction is regulated by dephosphorylation of L-CRMP4 as a direct consequence of glycogen synthase kinase $3 \beta$ (GSK3 $\beta$ ) phosphorylation and inactivation. GSK $3 \alpha$ and $\beta$ are serine/threonine kinases originally identified as regulatory kinases for glycogen synthase and subsequently implicated in signaling cascades downstream of Wnts, NGF (nerve growth factor), EGF (epidermal growth factor), semaphorins, and Hedgehog (Eickholt et al., 2002; Kockeritz et al., 2006). GSK3 has been widely studied as a potential therapeutic target for nerve regeneration and for a variety of diseases, including cancer and Alzheimer's disease (Kockeritz et al., 2006).

Here, we show that MAIs phosphorylate and inactivate GSK3 $\beta$, leading to subsequent CRMP4 dephosphorylation. We confirm previous reports that inhibition of GSK $3 \beta$ activity inhibits neurite outgrowth in cerebellar and dorsal root ganglion (DRG) neurons, mimicking the inhibitory effect of myelin, and demonstrate that the effects of GSK3 $\beta$ inhibitors are markedly attenuated by antagonizing CRMP4. We also demonstrate that overexpression of GSK3 $\beta$ attenuates myelindependent neurite outgrowth inhibition. We show that L-CRMP4 dephosphorylation enhances L-CRMP4 binding to RhoA and that a phospho-dependent change in L-CRMP4 conformation likely regulates this change in affinity. Together, these findings directly implicate GSK $3 \beta$ in the MAI signaling cascade and link the neurite outgrowth inhibitory effects of GSK3 $\beta$ inhibition to CRMP4. 


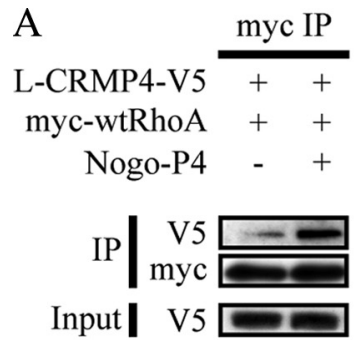

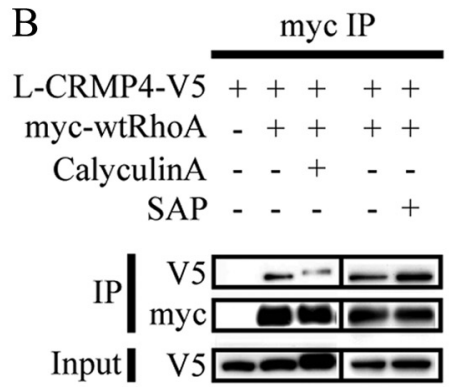

$\mathrm{C}$

myc IP

L-CRMP4-V5 wt wt wt wt AAA myc-RhoA - wt 188A wt wt

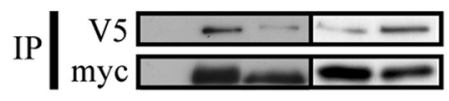

Input V5 $\longrightarrow$
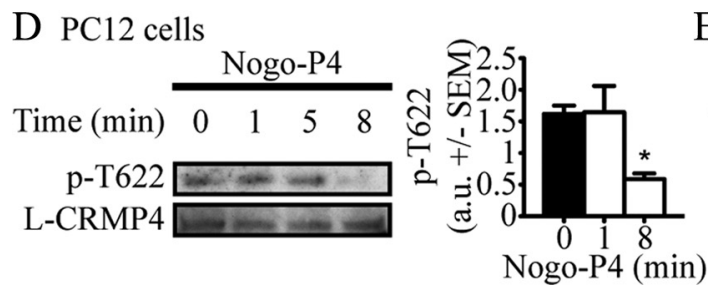

E Cerebellar Neurons

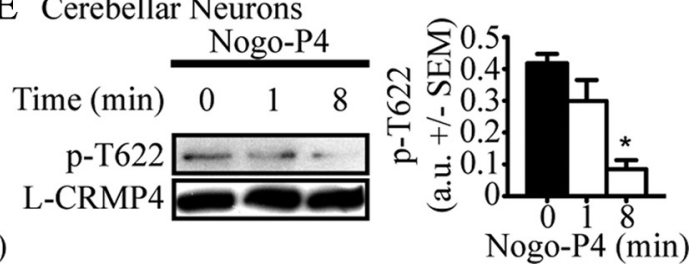

Figure 1. L-CRMP4 dephosphorylation is stimulated by Nogo-P4 and regulates L-CRMP4-RhoA binding. $A-C, P C 12$ cells $(\boldsymbol{A})$ or HEK293T cells ( $\boldsymbol{B}, \boldsymbol{C})$ were cotransfected with myc-RhoA constructs and L-CRMP4-V5 and subjected to immunoprecipitation with anti-myc antibody. $A$, PC12 cells were stimulated for 8 min with Nogo-P4 peptide. $\boldsymbol{B}$, Cells were treated for 30 min with $200 \mathrm{~nm}$ calyculin A before lysis or cell lysates were treated for 30 min with 10 USAP at $34^{\circ}$ C before immunoprecipitation. $\boldsymbol{D}, \boldsymbol{E}, \mathrm{PC} 12$ cells $(\boldsymbol{D})$ or cerebellar neurons infected with HSV-L-CRMP4-V5 (E) were stimulated for 1-8 min with Nogo-P4 peptide. Cell lysates were separated by SDS-PAGE and total and phosphorylated L-CRMP4 was detected by immunoblot. Quantifications in arbitrary units are from three experiments and are normalized for total L-CRMP4 loading. ${ }^{*} p<0.05$ by paired $t$ tests, two-tailed, compared with 0 min. a.u., Arbitrary units; IP, immunoprecipitation.

A PC12 cells

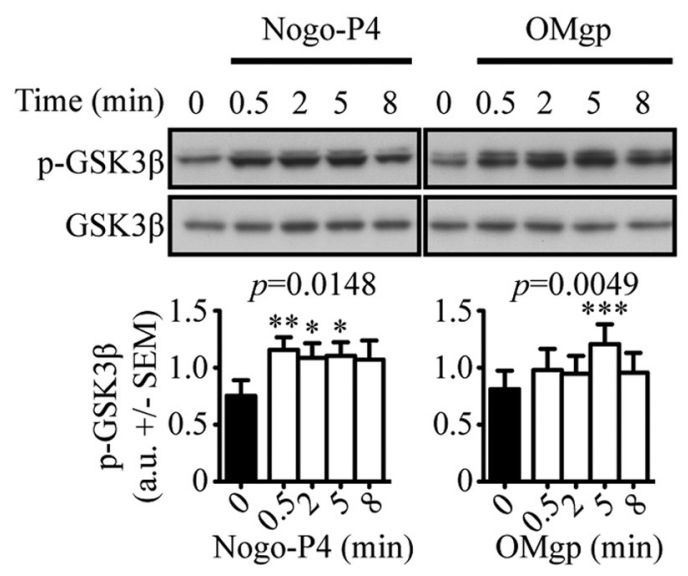

B Cerebellar Neurons

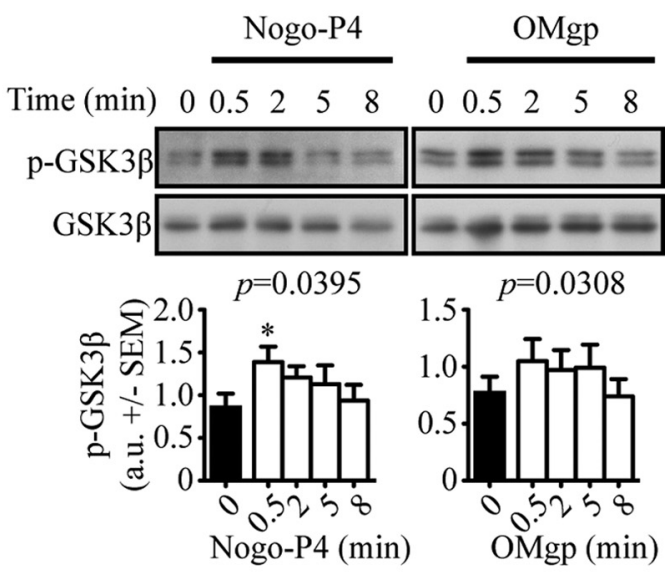

C PC12 cells

\begin{tabular}{|c|c|c|c|}
\hline \multirow[b]{2}{*}{ L-CRMP4-V5 } & \multicolumn{3}{|c|}{ V5 IP } \\
\hline & + & $+\quad+$ & + \\
\hline Nogo-P4 (min) & 0 & 80 & 8 \\
\hline GSK $3 \beta$ S9A & - & $-\quad+$ & + \\
\hline$\Leftrightarrow \mid \begin{array}{r}\mathrm{p}-\mathrm{T} 622 \\
\mathrm{~V} 5\end{array}$ & - & & \\
\hline
\end{tabular}

Figure 2. MAI-dependent phosphorylation of GSK3 $\beta$ results in L-CRMP4 dephosphorylation. $\boldsymbol{A}, \boldsymbol{B}, \mathrm{PC} 12$ cells $(\boldsymbol{A})$ or cerebellar neurons $(\boldsymbol{B})$ were stimulated for $0.5-8$ min with Nogo-P4 peptide or OMgp. Membrane fractions were separated by SDS-PAGE and total and phosphorylated GSK3 $\beta$ was detected by immunoblot. Quantifications are from at least seven experiments and are normalized for total GSK3 $\beta$ protein loading. ${ }^{*} p<0.05,{ }^{* *} p<0.01$, and ${ }^{* * *} p<0.001$ by Dunnett's post-test compared with 0 min following one-way RM ANOVA. ANOVA $p$ values are indicated above the graphs. C, PC12 cells were transiently transfected with L-CRMP4-V5 and GSK3 $359 \mathrm{~A}$ for $24 \mathrm{~h}$ and then treated with Nogo-P4 peptide for 8 min. L-CRMP4-V5 was immunoprecipitated and separated by SDS-PAGE, and total and phosphorylated L-CRMP4 was detected by immunoblot. a.u., Arbitrary units; IP, immunoprecipitation.

\section{Materials and Methods}

Plasmids and antibodies. CRMP4, C4RIP, and RhoA constructs were described previously (Alabed et al., 2007). CRMP4AAA was generated using a site-directed mutagenesis kit (Stratagene). The S188ARhoA construct was provided by Dr. Keith Burridge (University of North Carolina-Chapel Hill, Chapel Hill, NC) and GSK3 $\beta S 9 A$ by Dr. Dennis Stacey
(The Cleveland Clinic Foundation). L-CRMP4 antibody was generated by injecting rabbits with antigen RPGTTDQVPRQKYG as per the study by Quinn et al. (2003). Antiserum was affinity purified on an antigenSepharose column. Phospho-specific antibody that recognizes CRMP4b phosphorylated at Thr622 was generated in rabbit with the phosphopeptide FDLTT (pT)PKGGTPAGC (where pT is phosphothreonine). Anti- 
A

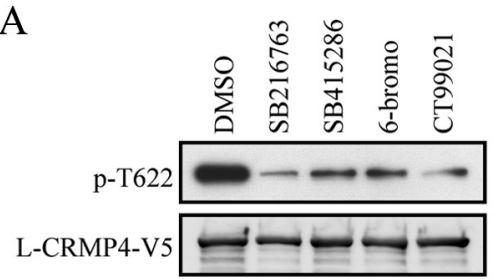

$\mathrm{B}$

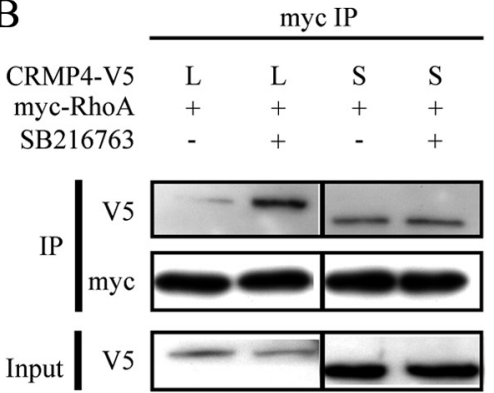

C Cerebellar Neurons DMSO
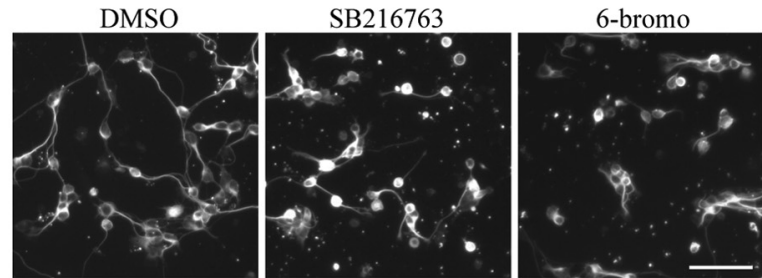

D DRG Neurons
DMSO

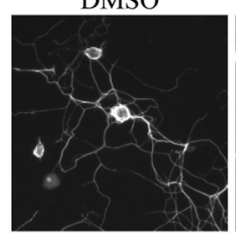

E Cerebellar Neurons

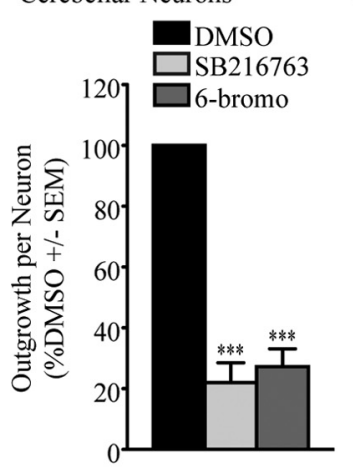

SB216763

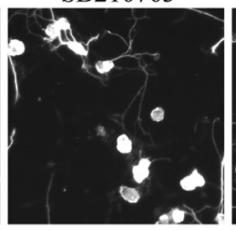

SB415286

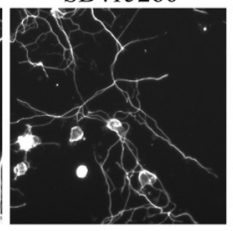

6-bromo

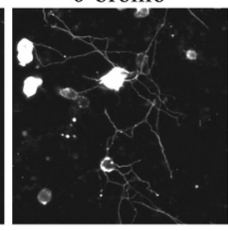

CT99021

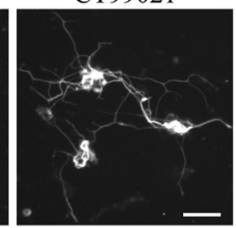

F DRG Neurons

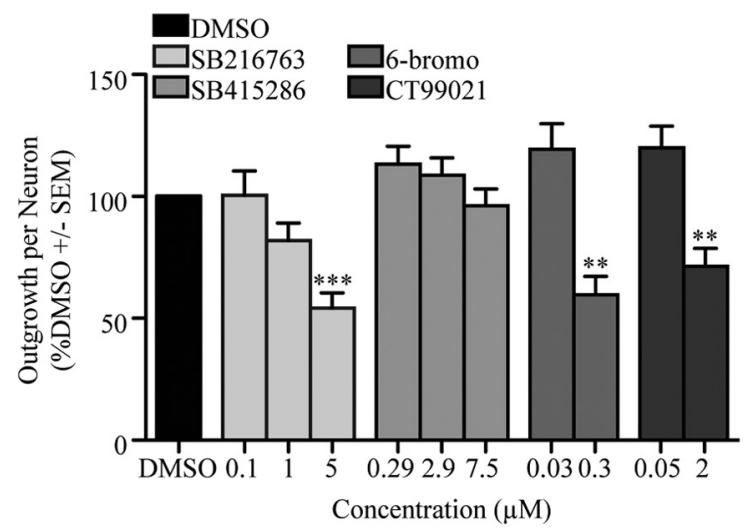

Figure 3. Pharmacologic inhibition of GSK3 $\beta$ enhances L-CRMP4-RhoA binding and inhibits neurite outgrowth. $A, H S V-L-$ CRMP4-V5-infected cerebellar neurons were treated with $5 \mu \mathrm{M}$ SB216763, $7.5 \mu \mathrm{M}$ SB415286, $300 \mathrm{~nm}$ 6-bromoindirubin-3'acetoxime, or $2 \mu \mathrm{m}$ CT99021 for $18 \mathrm{~h}$. Cell lysates were subjected to immunoprecipitation with anti-V5 antibody and analyzed by immunoblot with anti-pT622 to detect L-CRMP4 phosphorylation and anti-V5 to detect total L-CRMP4. Densitometry from two experiments shows a statistically significant reduction in phospho-L-CRMP4 signal normalized to total CRMP4; the one-way repeated-measures ANOVA $p$ value is 0.002 with Tukey's post-tests, indicating that values for all inhibitors are significantly different from those with DMSO ( $p<0.01)$. B, myc-RhoA and CRMP4-V5 constructs were transiently coexpressed in PC12 cells. To inhibit GSK3 $\beta$, cells were treated overnight with $5 \mu \mathrm{m}$ SB216763. Cell lysates were subjected to immunoprecipitation (IP) with anti-myc antibody and analyzed by immunoblot with anti-V5 or anti-myc antibodies. L, Long; S, short. C, D, Dissociated rat cerebellar neurons (C) or DRG neurons (D) were treated overnight with $5 \mu \mathrm{m}$ SB216763, 7.5 $\mu \mathrm{m}$ SB415286, $300 \mathrm{~nm}$ 6-bromoindirubin-3'-acetoxime, or $2 \mu \mathrm{M}$ CT99021, fixed, and stained with anti- $\beta$ III tubulin antibody to visualize neurite outgrowth. Scale bar, $50 \mu \mathrm{m}$. $\boldsymbol{E}, \boldsymbol{F}$, Quantification of cerebellar $(\boldsymbol{E})$ or DRG $(\boldsymbol{F})$ outgrowth/cell. Values are normalized to baseline outgrowth with DMSO vehicle control for each experiment. Determinations are from at least three experiments performed in duplicate. ${ }^{* *} p<0.01$ and ${ }^{* * *} p<0.001$ by Dunnett's post-tests following one-way RM ANOVA. 6-bromo, 6-Bromoindirubin-3'-acetoxime.

serum was affinity purified by depleting antibodies that recognize unphosphorylated CRMP4 on a nonphosphorylated peptide column followed by selecting phospho-specific antibodies on a phosphopeptide antigen column. Other antibodies used were mouse and rabbit anti-V5 and mouse anti-myc (Sigma-Aldrich), rabbit antiphosphothreonine (Invitrogen), rabbit antiphospho- and -total GSK3 $\beta$ (Cell Signaling Technology), mouse anti- $\beta$ III tubulin (Covance), and mouse anti-His (Qiagen).

Preparation of recombinant proteins. Stimulations to examine inhibitory responses were performed with Nogo-P4 peptide (Alpha Diagnostics), a 25 aa inhibitory peptide sequence (residues 31-55 of Nogo-66) sufficient to mediate the inhibitory properties of Nogo-66 (GrandPré et al., 2000), or His-tagged mouse OMgp (R\&D Systems) preclustered for $30 \mathrm{~min}$ at room temperature with mouse anti-His antibody. Myelin extracts (Igarashi et al., 1993; Hsieh et al., 2006) and GST-Nogo-66 were prepared as described previously (GrandPré et al., 2000).

Preparation of recombinant viruses. For herpes simplex virus (HSV) production, $\mathrm{pHSVPr}$ PUC plasmids were transfected into 2-2 Vero cells that were superinfected with $5 d l 1.2$ helper virus $1 \mathrm{~d}$ later. Recombinant virus was amplified through three passages and stored at $-80^{\circ} \mathrm{C}$ as described previously (Neve et al., 1997). Lentivirus particles were produced using a third-generation packaging system (Dull et al., 1998; Addgene) with GSK3 $\beta S 9 A-$ V5His cloned into the viral expression vector pRRLsinPPT. Recombinant viral particles were collected by high-speed centrifugation of supernatants from $293 \mathrm{~T}$ cells transfected with the expression vector and packaging mix by using Lipofectamine 2000.

CRMP-RhoA coimmunoprecipitation assay. HEK293T cells were grown to subconfluence and transfected with Lipofectamine 2000 according to the manufacturer's instructions (Invitrogen), washed twice with ice-cold PBS, and lysed in lysis buffer A containing the following (in mM): 50 Tris, $\mathrm{pH} 7.4,150 \mathrm{NaCl}, 1$ EDTA, $1 \%(\mathrm{v} / \mathrm{v})$ Triton X-100, $1 \mathrm{Na}_{3} \mathrm{VO}_{4}, 50 \mathrm{NaF}, 1$ PMSF, $100 \mathrm{~nm}$ calyculin A, and complete protease inhibitors (Roche Diagnostics). Lysates were precleared with protein A/G-agarose (Santa Cruz Biotechnology) and subjected to immunoprecipitation with myc-agarose or V5-agarose (Sigma). After washing three times with ice-cold lysis buffer, bound protein was eluted with SDS and immunoblotted with antiMyc or anti-V5. For time course experiments, PC12 cells were transfected for $24 \mathrm{~h}$ using Lipofectamine 2000 (Invitrogen) and differentiated with $50 \mathrm{ng} / \mathrm{ml} \mathrm{NGF}$ (Cedarlane Laboratories) for $24 \mathrm{~h}$. Cells were treated with Nogo-P4 peptide for the indicated period of time at $37^{\circ} \mathrm{C}$. Cells were then lysed, and proteins were immunoprecipitated as described above.

Assessment of protein phosphorylation. $\mathrm{PC} 12$ cells were differentiated in RPMI/1\% BSA/50 $\mathrm{ng} / \mathrm{ml}$ NGF for $24 \mathrm{~h}$ before treatment with recombinant proteins. P8 rat cerebellar neurons were prepared as previously described (Hsieh et al., 2006) and cultured in serum-free Sato's medium for $24 \mathrm{~h}$ before treatment. For L-CRMP4-V5-infected neurons, dissociated cerebellar neurons were cultured in the presence of virus for $4 \mathrm{~h}$ followed by serum starving for $20 \mathrm{~h}$.

Cell lysates were prepared by washing cells twice with ice-cold PBS and lysed in radioimmunoprecipitation assay buffer containing $20 \mathrm{~mm}$ 
HEPES, pH 7.4, $150 \mathrm{~mm} \mathrm{NaCl}, 1$ mм EDTA, $0.5 \%$ sodium deoxycholate, $0.1 \%$ SDS, $1 \%$ Triton X-100 with a protease inhibitor mixture (Roche) and phosphatase inhibitors ( $1 \mathrm{mM} \mathrm{Na}_{3} \mathrm{VO}_{4}, 50 \mathrm{~mm} \mathrm{NaF}, 100 \mathrm{~nm}$ calyculin A). Membrane fractionation was performed as described previously (Alabed et al., 2006). Lysates and membrane fractions were analyzed by SDS-PAGE and immunoblotting with antibodies recognizing phosphoand total CRMP 4 and/or GSK3 $\beta$. To quantify changes in protein phosphorylation, phospho-protein expression was assessed by densitometry and levels were normalized to the total level of the same protein in the lysate.

Neurite outgrowth assay. For outgrowth assays using pharmacologic inhibitors, SB216763, SB415286 (Sigma), 6-bromoindirubin-3'-acetoxime (EMD Biosciences), and CT99021 (generously provided by Dr. Rodolfo Marquez, School of Life Sciences, University of Dundee, Dundee, Scotland), were added to cultures after seeding. Dissociated embryonic day 13 (E13) chick and postnatal day 5 (P5) rat dorsal root ganglion (DRG) neurons were cultured in DRG medium (F-12 medium, $10 \% \mathrm{FBS}, 1 \%$ penicillin/streptomycin, $1 \%$ L-glutamine, $50 \mathrm{ng} / \mathrm{ml} \mathrm{NGF}$ ) in the presence of virus on poly-L-lysine- and laminin-coated substrates, fixed with $4 \%$ paraformaldehyde $/ 20 \%$ sucrose in PBS, and double stained with anti- $\beta$ III tubulin (Covance) and anti-V5 (Sigma) or antiHis (Qiagen) antibody. Dissociated cerebellar neurons were cultured in serum-free Sato's medium. Chick DRG neurite outgrowth lengths per cell were assessed using the NeuronJ plugin for ImageJ, a public domain JAVA image-processing program (http://rsb.info.nih.gov/ij/), as described previously (Fournier et al., 2003). Rat DRG and cerebellar neuron outgrowth was analyzed with the neurite outgrowth module of MetaXpress. For rat DRG cultures infected with lentiviruses, the neurons expressing the constructs were identified using the multiwavelength cellscoring module of MetaXpress and the length of the neurites from only the expressing cells was measured using the NeuronJ plugin for ImageJ.

Densitometry and statistical analysis. Densitometry was performed using Adobe Photoshop and all quantifications were normalized for total protein loading. Statistical analysis was performed using GraphPad Prism and the specific tests used are indicated in the text or in the figure legends.

\section{Results}

L-CRMP4-RhoA binding is regulated by

MAI-dependent dephosphorylation

As reported previously, the association between RhoA and L-CRMP4 is enhanced by stimulation with Nogo-P4 peptide, an inhibitory fragment of Nogo-A (GrandPré et al., 2000), in transfected PC12 cells (Fig. 1 A) and cerebellar neurons (Alabed et al., 2007). The rapid enhancement of this protein-protein interaction led us to investigate the potential regulatory role of protein phosphorylation on this process. In 293T cells transfected with myc-wild-type (wt)-RhoA and L-CRMP4-V5, myc immunoprecipitates contain L-CRMP4-V5 (Fig. $1 \mathrm{~B}$ ). Treatment of transfected 293T cells with the serine/threonine phosphatase inhibitor calyculin A causes an upward mobility shift of L-CRMP4-V5 indicative of L-CRMP4 phosphorylation. While there is no apparent mobility shift in wt-RhoA following calyculin A treatment, this does not exclude the possibility that RhoA is also phosphorylated. Calyculin A treatment diminishes the L-CRMP4wt-RhoA coimmunoprecipitation, demonstrating that phosphorylation of L-CRMP4 and/or RhoA disrupts their binding. When 293T cell lysates are treated with shrimp alkaline phosphatase (SAP) to stimulate protein dephosphorylation, the association between L-CRMP4-V5 and myc-wt-RhoA is enhanced, similar to the effect of Nogo treatment. We then asked whether dephosphorylation of RhoA and/or L-CRMP4 is capable of enhancing RhoA-L-CRMP4 binding. The binding properties of a RhoA mutant with the phospho-residue serine 188 mutated to alanine (nonphosphorylatable S188ARhoA) and of an L-CRMP4 triple alanine substitution mutant (L-CRMP4-AAA) for the three carboxy terminal phospho-residues targeted by GSK3 $\beta$ (Thr622, Thr627, Ser631) were assessed. RhoAS188A binds more weakly than wt-RhoA to
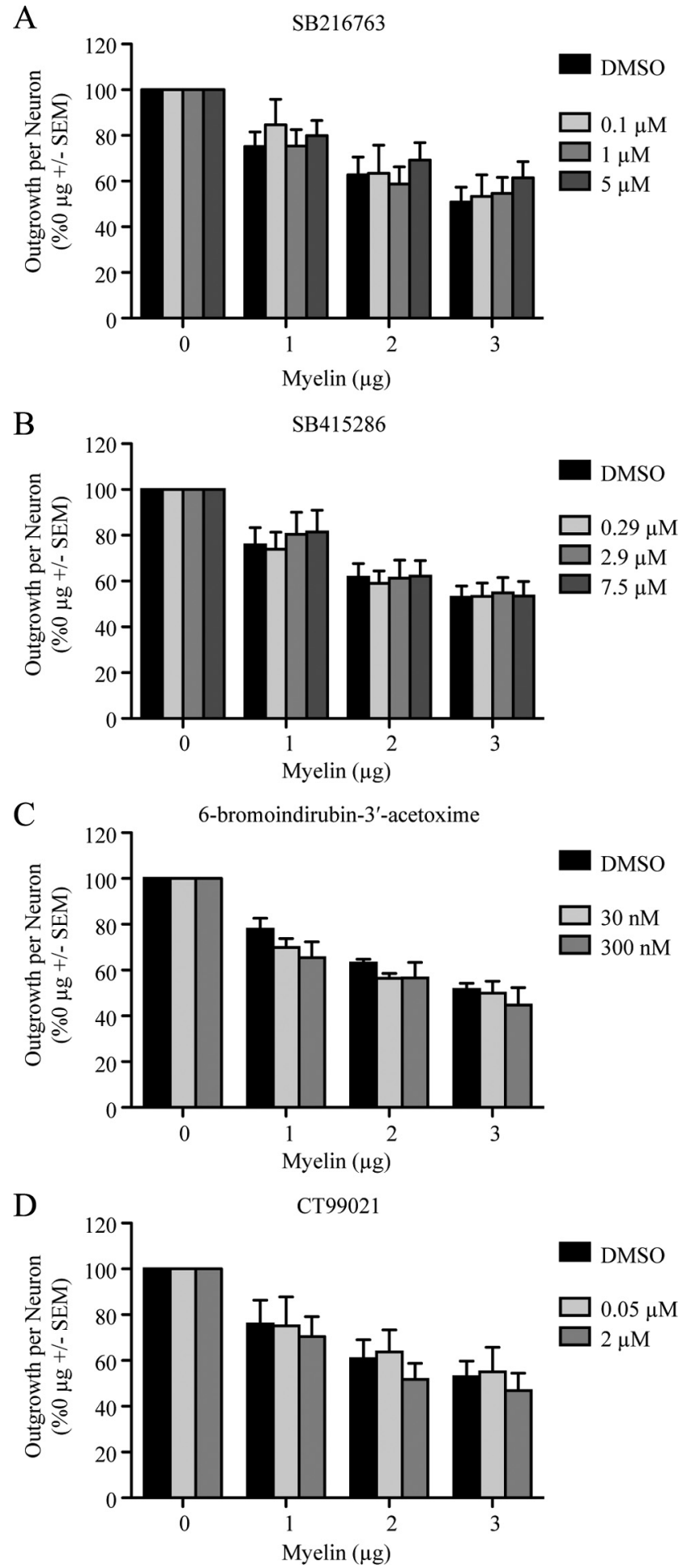

Figure 4. Effects of myelin and GSK3 $\beta$ inhibitors on neurite outgrowth inhibition are not additive. $A-D$, Quantification of DRG neurite outgrowth/cell from neurons plated on myelin and treated with vehicle control (DMSO) and increasing doses of SB216763 (A), SB415286 (B), 6-bromoindirubin-3' acetoxime (C), or CT99021 (D). Values are normalized to outgrowth with no myelin. Determinations are from at least four experiments performed in duplicate. For DMSO-treated neurons, $p<0.001$ for all doses of myelin compared with no myelin by Dunnett's post-tests following one-way RM ANOVA. Two-way ANOVA shows no significant differences in outgrowth on myelin between cultures treated with DMSO and those treated with any GSK inhibitor.

wt-L-CRMP4 (Fig. 1C). However, L-CRMP4-AAA binds more strongly than wt-L-CRMP4 to wt-RhoA (Fig. 1C). Together, these findings indicate that dephosphorylation of L-CRMP4 favors L-CRMP4-RhoA binding as does Nogo stimulation. 
A

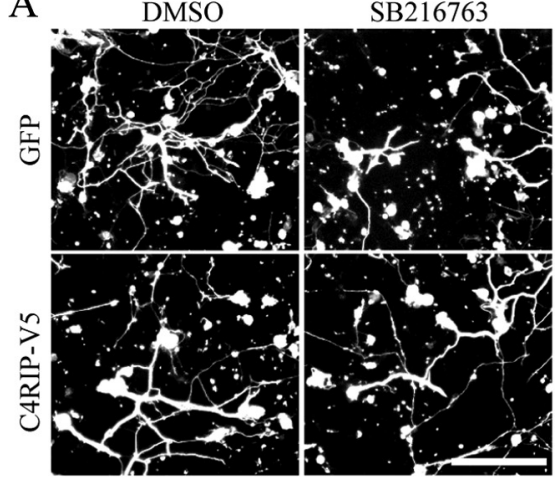

B

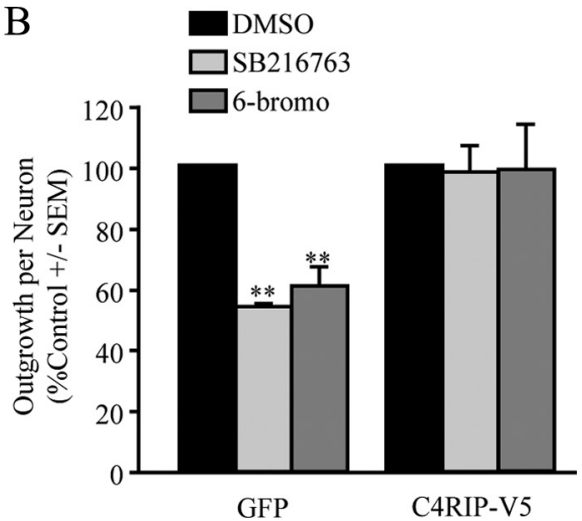

Figure 5. Pharmacologic inhibition of GSK3 $\beta$ inhibits neurite outgrowth in an L-CRMP4-dependent manner. $\boldsymbol{A}, \mathrm{HSV}$-GFP- or HSV-C4RIP-infected dissociated DRG neurons were grown overnight in the presence of $5 \mu \mathrm{M}$ SB216763, fixed, and stained with anti- $\beta$-III tubulin antibody. Scale bar, $100 \mu \mathrm{m}$. B, Quantification of DRG neurite outgrowth/cell from HSV-GFP- or HSV-C4RIPinfected neurons treated overnight with $5 \mu \mathrm{m}$ SB216763 or 300 nм 6-bromoindirubin-3'-acetoxime (6-bromo). Values are normalized to baseline outgrowth with DMSO vehicle control. Determinations are from three experiments performed in duplicate. ${ }^{* *} p<0.01$ by Student's $t$ test compared with DMSO.

To evaluate the effect of Nogo stimulation on L-CRMP4 phosphorylation, PC12 cells or L-CRMP4-V5-infected cerebellar neurons were treated with Nogo-P4 peptide and L-CRMP4 phosphorylation was assessed by Western blotting with a phospho-specific antibody recognizing pThr622 of L-CRMP4 [corresponding to Thr 509 of S-CRMP4 (Cole et al., 2004b)]. Nogo-P4 stimulation diminishes L-CRMP4 phosphorylation in both PC12 cells (Fig. 1D) and cerebellar neurons (Fig. 1E).

\section{L-CRMP4 is dephosphorylated in a GSK3 $\beta$-dependent manner in response to MAIs}

Dephosphorylation of L-CRMP4 suggests engagement of a CRMP4-directed phosphatase and/or inactivation of an L-CRMP4directed kinase in response to MAIs. L-CRMP4 phosphorylation is sequentially regulated by GSK $3 \beta$ on residues Ser631, Thr627, and Thr622 following a priming phosphorylation event that may be mediated by DYRK2 (Cole et al., 2004b). Inactivation of GSK3 $\beta$ by phosphorylation on Ser9 leads to a rapid decrease in phospho content of its substrates. GSK3 $\beta$ phosphorylation and inactivation are an important regulatory step in response to many factors including NGF and Wnt (Cohen and Frame, 2001; Zhou et al., 2004 ); therefore, we assessed the role of GSK3 $\beta$ in Nogo signaling. We find that GSK $3 \beta$ is phosphorylated in membrane fractions from Nogo-P4- or OMgp-stimulated PC12 cells (Fig. 2A) and cerebellar neurons (Fig. $2 \mathrm{~B}$ ). To examine the subcellular distribution of inactive GSK, we performed immunostaining and observed an increase in phospho-GSK in the central domain of growth cones undergoing collapse in response to both Nogo-P4 and OMgp (supplemental Fig. 1, available at www. jneurosci.org as supplemental material). To test whether GSK $3 \beta$ phosphorylation and inactivation lead to L-CRMP4 dephosphorylation, we overexpressed a constitutively active form of GSK3 $\beta$ (GSK3 $\beta S 9 A$ ) and examined the effect of Nogo on L-CRMP4 phosphorylation. Overexpression of GSK3 $\beta S 9 A$ blocks the Nogo-P4-dependent decrease in L-CRMP4 dephosphorylation, indicating that L-CRMP4 dephosphorylation is GSK3 $\beta$ dependent (Fig. 2C).

\section{Inactivation of GSK3 $\beta$ inhibits neurite outgrowth in an} L-CRMP4-dependent manner

Our data support a model whereby Nogo induces GSK3 $\beta$ inactivation, resulting in L-CRMP4 dephosphorylation and enhanced

L-CRMP4-RhoA complex formation. If this is the case, then GSK $3 \beta$ inactivation should diminish CRMP4 phosphorylation, increase L-CRMP4 association with RhoA, and inhibit neurite outgrowth. To test this, levels of L-CRMP4 phosphorylation were assessed in PC12 cells treated with GSK3 inhibitors. As expected for a known GSK3 $\beta$ substrate (Cole et al., 2004b), phospho-LCRMP4 levels were dramatically reduced in cells treated with the GSK3 inhibitors SB216763, SB415286, 6-bromoindirubin3' -acetoxime, and CT99021 (Fig. 3A) [reductions in phospho-L-CRMP4 of $94 \pm 4$, $76 \pm 9,78 \pm 15$, and $90 \pm 6 \%( \pm$ SEM $)$, respectively, normalized to total L-CRMP4 loading, $n=2$ ]. Overnight stimulation of PC12 cells with SB216763 (Fig. 3B) or 6-bromoindirubin- 3 '-acetoxime (data not shown) increases the association of RhoA with L-CRMP4 but not S-CRMP4. The specific effect of the pharmacologic GSK3 $\beta$ inhibitors on the long isoform of CRMP 4 mimics that of Nogo treatment (Alabed et al., 2007). Previous reports have shown that strong GSK3 inhibition reduces neurite outgrowth (Kim et al., 2006). Consistent with this, we find that treatment of rat cerebellar neurons (Fig. 3C,E) or DRG neurons (Fig. 3D,F) with several GSK3 inhibitors diminishes neurite outgrowth. Weak GSK3 inhibition has previously been shown to promote branching of immature hippocampal and DRG neurons (Kim et al., 2006) but to have no significant effect on branching in later stage neurons (Dill et al., 2008). Consistent with this, we observed no increase in the number of primary processes or branches with low doses of GSK inhibitors in postnatal rat DRGs (supplemental Fig. 2, available at www.jneurosci.org as supplemental material). The decrease in branching observed at high doses of GSK inhibitors is likely due to the decrease in the overall growth of these neurons (Fig. 3F). In our hands, every GSK3 inhibitor tested inhibits neurite outgrowth except for SB415286 (Fig. 3F), raising the possibility that the known SB415286 effects on additional kinases (Bain et al., 2007) may neutralize the neurite outgrowth inhibitory effect of GSK3 $\beta$ inhibition.

To test whether myelin and GSK3 inhibition has an additive effect on neurite outgrowth inhibition, we examined neurite outgrowth from rat DRG neurons with combined exposure to myelin and GSK3 inhibitors. SB216763, 6-bromoindirubin-3'-acetoxime, and CT99021 inhibit neurite outgrowth in a dose-dependent fashion (Fig. 3C-F) but do not enhance myelin-dependent inhibition (Fig. $4 A-D$ ) or inhibition by a purified GST-Nogo66 substrate (supplemental Fig. 3, available at www.jneurosci.org as supplemental material) further supporting our data that GSK3 is part of the myelin signaling pathway leading to neurite outgrowth inhibition.

To determine whether the reduced neurite outgrowth that accompanies GSK3 inhibition requires L-CRMP4, we assessed the effects of C4RIP (CRMP4-RhoA Interfering Peptide), an antagonist of L-CRMP4-RhoA binding (Alabed et al., 2007). Remarkably, the neurite outgrowth inhibitory effect induced by GSK3 inhibition is dramatically attenuated by infecting neurons with HSV-C4RIP (Fig. 5). This indicates that the neurite outgrowth inhibition induced by GSK3 inhibitors requires L-CRMP4. 
Overexpression of GSK3 $\beta$ attenuates myelin-dependent inhibition

A second prediction from our biochemical data is that overexpression of GSK $3 \beta$ would overcome myelin inhibition by diminishing binding between RhoA and phosphorylated L-CRMP4. Overexpression of GSK3 $\beta$ enhances the phosphorylation of both S-CRMP4 and L-CRMP4 (Fig. 6A) but specifically reduces binding between RhoA and the long isoform of CRMP4 (Fig. 6B). Further, DRG neurons infected with a GSK3 $\beta$ S9A lentivirus (Fig. 6C) grow significantly better on an inhibitory myelin or GST-Nogo66 substrate (Fig. $6 C, D$ ), demonstrating that GSK3 $\beta$ inactivation is necessary for myelin inhibition.

A phospho-dependent conformation of L-CRMP4 affects its binding properties Regulation of GSK $3 \beta$ affects phosphorylation of S-CRMP4 and L-CRMP4, yet only the L-CRMP4 isoform demonstrates GSK3 $\beta$ - or Nogo-regulated RhoA binding (Alabed et al., 2007) (Figs. 3, 6). Further, RhoA binds more robustly to L-CRMP4 than S-CRMP4 (Alabed et al., 2007). We therefore considered the possibility that RhoA binds to L-CRMP4 on two distinct binding sites, one in the carboxy terminal region that is shared by S-CRMP4 and L-CRMP4 and one in the unique N-terminal portion of L-CRMP4. We assessed the ability of individual L-CRMP4 domains to interact with RhoA by coimmunoprecipitation from transfected 293T cells (Fig. 7A,B). We detected a binding site for RhoA in the common dihydropyrimidinase (DHP) region of CRMP4 but failed to detect binding between RhoA and the unique N-terminal domain of L-CRMP4, C4RIP (Fig. 7B).

We next considered the possibility that L-CRMP4 may exist in a phospho-dependent conformation that regulates binding to RhoA. To test this possibility, we examined binding between RhoA and the triple alanine substitution mutant of L-CRMP4 or S-CRMP4. L-CRMP4 AAA binds to RhoA more strongly than wt-LCRMP4; however, binding between RhoA and S-CRMP4 AAA is indistinguishable from RhoA binding to wt-S-CRMP4 (Fig. 7C). This suggests that the phosphorylation status of the carboxy terminus of L-CRMP4 affects a RhoA binding site that is dependent on the unique $\mathrm{N}$ terminus of L-CRMP4. This could be ascribed to a folded conformation that stabilizes a single RhoA binding site or to a generation of a new conformationdependent RhoA binding site in the unique $\mathrm{N}$ terminus of L-CRMP4 (Fig. 7D). Importantly, we find that in PC12 cells, stimulation with Nogo-P4 fails to further enhance binding between L-CRMP4 AAA and RhoA (Fig. 7E) demonstrating

A

C

D

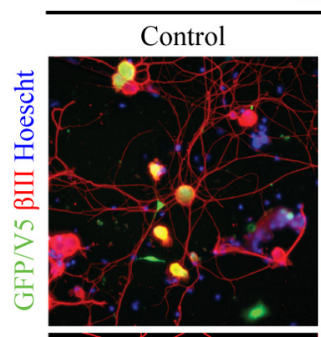

GFP
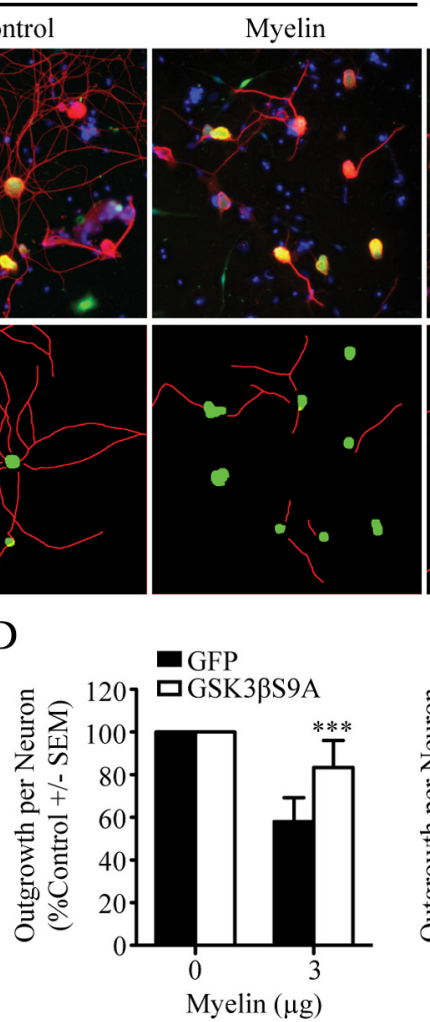

Figure 6. GSK3 $\beta$ overexpression attenuates myelin inhibition. $A$, Lysates from 293T cells transfected with GSK3 $\beta$ and CRMP4 -V5 constructs were subjected to immunoprecipitation with an anti-V5 antibody and probed with anti-phosphothreonine or anti-V5 antibody. $\boldsymbol{B}$, Lysates from $293 \mathrm{~T}$ cells transfected with myc-RhoA, GSK3 $\beta$, and CRMP4-V5 constructs were subjected to immunoprecipitation with an anti-myc antibody and probed with anti-V5, anti-myc, and anti-GSK3 $\beta$ antibodies. C, HSV-GFP- or GSK3 $\beta$ S9A-V5-infected dissociated DRGs were grown overnight on myelin, fixed, and stained with anti- $\beta$-III tubulin antibody (red) and Hoescht nuclear stain (blue). The green image reflects GFP signal from the control virus or anti-V5 staining detecting GSK3 $\beta$ S9A overexpression. Skeletonized output images of HSV-GFP- or GSK3 $\beta S 9 A-V 5$-positive neuronal cell bodies (green) and their associated neurites (red) used for quantification are shown in the bottom row. Scale bar, $50 \mu \mathrm{m}$. D, Quantification of DRG neurite outgrowth/cell from HSV-GFP- or GSK3 $\beta$ S9A-infected DRG neurons grown on myelin or GST-Nogo-66 substrates. Determinations are from three experiments. ${ }^{*} p<0.05$ and ${ }^{* * *} p<0.001$ by Bonferroni's post-tests following two-way RM ANOVA. IP, Immunoprecipitation; L, long; $S$, short. that Nogo-dependent dephosphorylation of L-CRMP4 is responsible for enhancing L-CRMP4-RhoA binding. Finally, we infected DRG neurons with recombinant HSV-L-CRMP4 AAA and assessed neurite outgrowth. We find that overexpression of L-CRMP4 AAA alone modestly but significantly inhibits neurite outgrowth $(15.1 \pm 3.48 \%$ reduction \pm SEM, $p=0.049$ by two-tailed paired $t$ test, $n=3$ ) indicating that dephosphorylation of CRMP4 alone is sufficient to mediate some neurite outgrowth inhibition; however, dephosphoryla- 
A

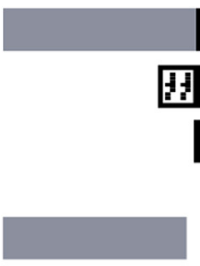

B

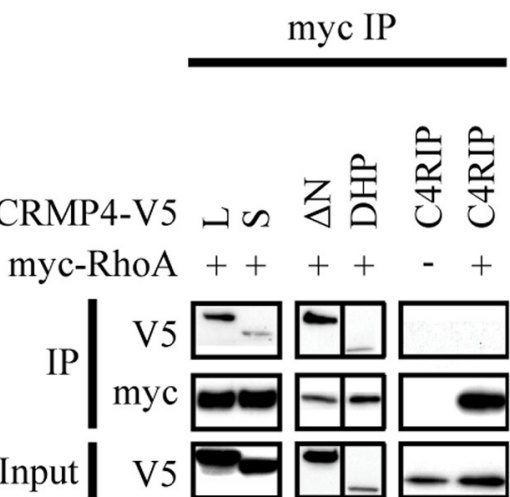

$\mathrm{D}$
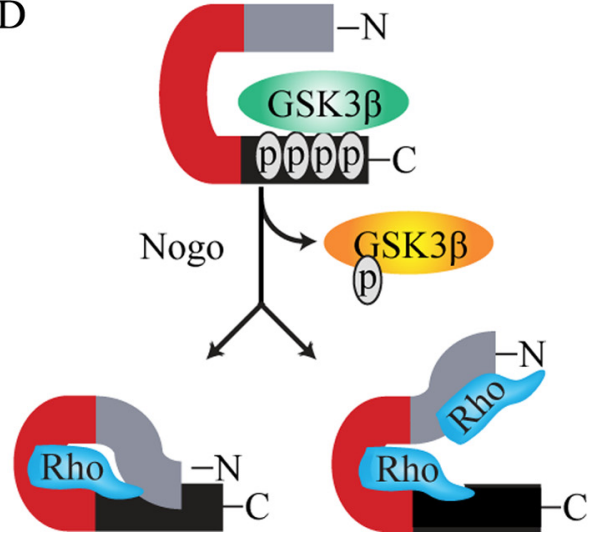
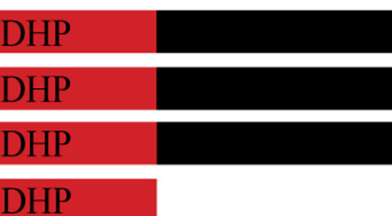

DHP

C

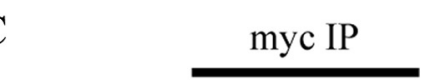

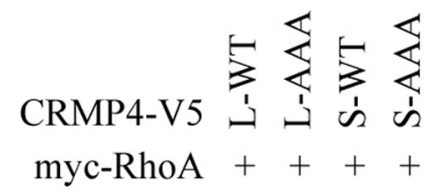

$$
\text { IP }
$$

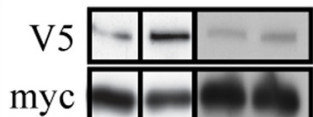

Input

V5

$\mathrm{E}$
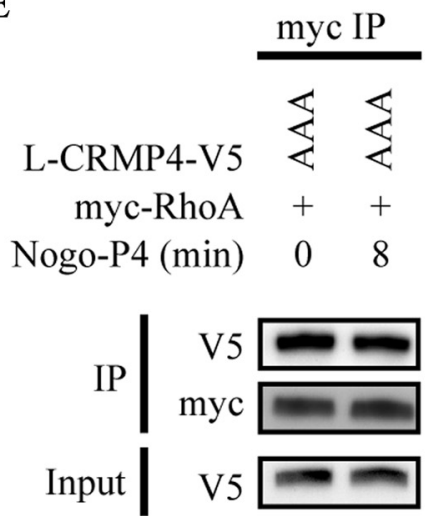

Figure 7. L-CRMP4 conformation is phospho dependent and regulates RhoA recruitment. $A$, Schematic representation of full-length and mutant CRMP4 constructs. B, C, HEK293T cells were transiently cotransfected with myc-RhoA and CRMP-V5 constructs. Lysates were subjected to immunoprecipitation with anti-myc antibody and immunoblotted with anti-myc or anti-V5 antibodies. D, A model of how L-CRMP4 binding to RhoA may be regulated by a phosphorylation-dependent conformational change in L-CRMP4. Dephosphorylation of the carboxy terminal region of L-CRMP4 may enhance the folding interaction between the amino and carboxy termini of the protein and modify the protein conformation to favor binding to RhoA or may change the conformation of the $\mathrm{N}$ terminus of $\mathrm{L}-\mathrm{CRMP} 4$, exposing a second RhoA binding site. $E$, $\mathrm{PC} 12$ cells were transiently cotransfected with myc-RhoA and CRMP4 AAA-V5. Cells were stimulated with Nogo-P4 peptide and lysates were subjected to immunoprecipitation with anti-myc antibody and immunoblotted with anti-myc or anti-V5 antibodies. IP, Immunoprecipitation; L, long; S, short.

tion of L-CRMP4 in combination with RhoA activation mediates more robust inhibitory effects.

\section{Discussion}

We find that MAIs induce phosphorylation and inactivation of GSK3 $\beta$, which regulate CRMP4 phosphorylation and binding to RhoA (supplemental Fig. 4, available at www.jneurosci.org as supplemental material). GSK3 $\beta$ inhibition mimics the effect of myelin on neurite outgrowth and this requires CRMP4. We also demonstrate that GSK3 $\beta$ inactivation is necessary for MAI signaling because overexpression of active GSK3 $\beta$ attenuates MAI-dependent neurite outgrowth inhibition. Together, these findings provide novel insights into the neuronal mechanism of action of GSK3 $\beta$ and suggest additional molecular targets to promote neuronal repair following CNS injury.
MAI-dependent regulation of GSK3 $\beta$

The molecular links between cell surface MAI receptors and RhoA regulation of the cytoskeleton have not been fully elucidated. We have previously implicated an L-CRMP4-RhoA interaction in this pathway and have now demonstrated that this interaction is negatively regulated through L-CRMP4 phosphorylation by GSK3 $\beta$. The kinase responsible for GSK3 $\beta$ phosphorylation in response to MAI stimulation remains to be determined. PKC is an intriguing candidate because it is activated by MAIs and blockade of PKC attenuates myelin-dependent inhibition (Sivasankaran et al., 2004). GSK3 $\beta$-mediated phosphorylation of the $\mathrm{C}$ terminus of L-CRMP4 is also dependent on priming phosphorylation at Ser635. Although both CDK5 and DYRK2 prime CRMP4 in vitro, the in vivo priming kinase is undetermined (Cole et al., 2006). Whether the priming kinases are directly regulated in response to MAI stimulation remains unknown.

\section{Neurite outgrowth inhibition and GSK3 $\beta$ inactivation}

We provide the first example of a neurite outgrowth inhibitory ligand that stimulates phosphorylation and inactivation of GSK $3 \beta$. Our findings are consistent with several reports demonstrating that pharmacologic inhibition of GSK3 inhibits neurite outgrowth, but differ from those reporting promotion of axon branching with GSK3 inhibition (Owen and GordonWeeks, 2003; Shi et al., 2004; Kim et al., 2006). In an elegant study to examine why GSK3 inhibition can both enhance branching and inhibit outgrowth, Kim et al. (2006) have described a correlation between GSK3 activity toward primed or nonprimed substrates and neuronal phenotypes. Specifically, introduction of a GSK3 $\beta$ mutant that selectively phosphorylates nonprimed substrates (GSK3 $\beta$ R96A) results in reduced axon branching. Further, low concentrations of GSK3 inhibitors that increase axon branching mainly diminish the phosphorylation of primed GSK3 substrates. GSK3 $\beta$ regulates L-CRMP4 phosphorylation on priming-dependent and -independent residues (Y. Z. Alabed and A. E. Fournier, unpublished data) and these sites may be differentially affected by various concentrations of GSK3 $\beta$ inhibitors. MAI-dependent inactivation of GSK3 $\beta$ may impact additional priming-independent substrates, leading to neurite outgrowth inhibition; however, this is difficult to reconcile with the ability of C4RIP to reverse myelin- and SB216763-dependent outgrowth inhibition.

\section{Spatial targeting of GSK $3 \beta$}

MAI effects on GSK3 $\beta$ phosphorylation were variable in wholecell lysates but consistent in membrane fractions. This suggests that a specific pool of GSK3 $\beta$ may be regulated in response to MAIs. A commonly accepted view is that GSK3 may be regulated 
at discrete sites within the axon and growth cone to target specific substrates. The engagement of distinct spatially segregated pools of target substrates could explain how inhibitory MAIs and growth-promoting neurotrophins both phosphorylate and inactivate GSK3 $\beta$ (Zhou et al., 2004).

\section{GSK3 $\beta$ as a therapeutic target}

Based on findings indicating growth-promoting and neuroprotective effects of GSK3 $\beta$ inhibition, clinical studies in spinal cord injury using stem cells and the GSK $3 \beta$ inhibitor lithium are being pursued (Cyranoski, 2007). Our findings demonstrate that robust GSK $3 \beta$ inhibition impedes axon extension, raising concerns regarding the efficacy of such a treatment. A recent study has demonstrated that lithium and SB415286 increase neurite outgrowth on myelin and CSPG substrates and stimulate growth of corticospinal tract fibers around the site of a spinal cord injury (Dill et al., 2008). We do not detect enhanced outgrowth of SB415286-treated DRG neurons on myelin substrates (Fig. 4) and this drug does not inhibit neurite outgrowth on a laminin substrate while other GSK3 $\beta$ inhibitors do (Fig. $3 F$ ). The offtarget effects of lithium and SB415286 (Bain et al., 2003, 2007) raise the possibility that the effects of these drugs on neurite outgrowth are not through GSK3 $\beta$. We are confident that the neurite outgrowth inhibitory effects described here are attributable to GSK3 $\beta$, since CT9902 1 is a very specific GSK3 $\beta$ inhibitor. The only other identified substrate for CT99021 is CDK2CyclinA, but this substrate is strongly targeted by SB415286, which does not inhibit neurite outgrowth (Bain et al., 2007). The in vitro inhibition of outgrowth does not, however, preclude the possibility that the doses used in vivo elicit an axonal sprouting phenotype.

\section{L-CRMP4 and neurite outgrowth inhibition}

Our data suggest that overexpression of GSK3 $\beta$ inhibits formation of an L-CRMP4-RhoA complex and may be protective in the context of myelin inhibition. The partial nature of the rescue is likely explained by exposure of the neurons to the inhibitory substrate during the delay between lentiviral transduction and expression of GSK3 $359 \mathrm{~A}$, but it is also possible that alternative parallel pathways are involved in myelin inhibition of outgrowth. The previously reported proapoptotic function of GSK3 $\beta$ (Zhou et al., 2004) makes its overexpression an unlikely route for therapeutics, highlighting the importance of understanding its targets for promoting outgrowth on myelin. GSK3 regulates the phosphorylation and activation of many microtubule-associated proteins, including APC, CRMP2, CRMP4, MAP1b, MAP2, NF, Tau, and kinesin light chain, which would be affected in an overexpression paradigm (Cole et al., 2004a; Zhou and Snider, 2005). CRMP2 is phosphorylated in a ROCK (Rho-associated kinase)dependent manner during Nogo or MAG signaling and may contribute to neurite outgrowth inhibition via dysregulated microtubule dynamics (Mimura et al., 2006). While CRMP4 is capable of binding to microtubules (Fukata et al., 2002), it is not a known ROCK substrate and its in vivo function likely differs from CRMP2 for several reasons. First, overexpression of S-CRMP4 in hippocampal neurons or SHSY5Y cells has a modest effect on axon outgrowth when compared with the robust elongation effect of S-CRMP2 (Cole et al., 2004a). Second, L-CRMP4 colocalizes with SV2-positive vesicles and binds to the endocytic adaptor protein intersectin, suggesting a role in endocytosis (Quinn et al., 2003). Third, L-CRMP4 overexpression promotes an actin-based phenotype in DRG neurons promoting the extension of filopodia and neurite branches (Alabed et al., 2007). This actin-based phe- notype is consistent with the ability of CRMP4 to bundle F-actin (Rosslenbroich et al., 2005) and to bind to RhoA (Alabed et al., 2007). Overexpression of a splice variant of CRMP1 together with CRMP2 antagonizes Rho signaling (Leung et al., 2002) and overexpression of CRMP2 can switch RhoA- and Rac-1-dependent morphological changes in N1E-115 cells (Hall et al., 2001). However, CRMP4 siRNA treatment does not affect levels of phosphoLIMK or phospho-cofilin (M. Pool and A. E. Fournier, unpublished data), nor does it affect neurite outgrowth on laminin substrates (Alabed et al., 2007), indicating that CRMP4 does not directly regulate signaling downstream of RhoA. Further, the modest inhibitory effect of L-CRMP4 AAA expression on neurite outgrowth suggests that dephosphorylated CRMP4 and active RhoA cooperate to mediate neurite outgrowth inhibition, perhaps by regulating the localized formation of a signaling complex. How RhoA phosphorylation may be regulated to modulate MAI signaling and binding to CRMP4 is also an open question, since RhoAS188A binds more weakly to CRMP4 (Fig. 1C). Finally, the long isoforms of CRMPs can serve different functions from the short isoforms, perhaps even serving as short-isoform antagonists (Yuasa-Kawada et al., 2003). The ability of C4RIP to inhibit L-CRMP4-RhoA binding and to attenuate Nogo- and SB216763-dependent outgrowth inhibition suggests that the role of dephosphorylated L-CRMP4 in mediating neurite outgrowth inhibition may be linked to its ability to bind to RhoA and is suggestive of an actin-dependent phenotype.

\section{CRMP4 structure}

The crystal structures of murine CRMP1 (Deo et al., 2004) and human CRMP2 (Stenmark et al., 2007) have been solved, but the structures do not include the N-terminal extension of the long isoforms or the carboxy terminal region containing the GSK3 $\beta$ target residues. The lack of structural data for the carboxy termini is a function of proteolytic susceptibility of this region (Deo et al., 2004). Our findings suggest that full-length L-CRMP isoforms may undergo a fold resulting in a phospho-dependent conformation that regulates additional protein-protein interactions. For simplicity, our model is presented with a single CRMP molecule; however, it is known that CRMPs form heterotetramers (Wang and Strittmatter, 1997). It is possible that intermolecular binding of RhoA to the N terminus of one L-CRMP4 molecule and the DHP region of a second molecule may occur. Further, it is possible that phosphorylation of L-CRMP4 in the carboxy terminus may affect the oligomerization properties of L-CRMP4 and that RhoA may favor binding to L-CRMP4 monomers or oligomers. Additional interactions conferred by phospho-dependent conformational changes in L-CRMP4 could play a key role in CRMP function by regulating binding affinities to upstream regulators such as GSK3 and/or to potential effectors such as RhoA. A better understanding of the impact of phosphorylation on L-CRMP4 binding interactions will likely yield additional insights into L-CRMP4 function and into intracellular mechanisms regulating neurite outgrowth inhibition.

\section{References}

Alabed YZ, Grados-Munro E, Ferraro GB, Hsieh SH, Fournier AE (2006) Neuronal responses to myelin are mediated by ROCK. J Neurochem 96:1616-1625.

Alabed YZ, Pool M, Ong Tone S, Fournier AE (2007) Identification of CRMP4 as a convergent regulator of axon outgrowth inhibition. J Neurosci 27:1702-1711.

Bain J, McLauchlan H, Elliott M, Cohen P (2003) The specificities of protein kinase inhibitors: an update. Biochem J 371:199-204.

Bain J, Plater L, Elliott M, Shpiro N, Hastie CJ, McLauchlan H, Klevernic I, Arthur JS, Alessi DR, Cohen P (2007) The selectivity of protein kinase inhibitors: a further update. Biochem J 408:297-315. 
Byk T, Dobransky T, Cifuentes-Diaz C, Sobel A (1996) Identification and molecular characterization of Unc-33-like phosphoprotein (Ulip), a putative mammalian homolog of the axonal guidance-associated unc-33 gene product. J Neurosci 16:688-701.

Cohen P, Frame S (2001) The renaissance of GSK3. Nat Rev Mol Cell Biol 2:769-776.

Cole A, Frame S, Cohen P (2004a) Further evidence that the tyrosine phosphorylation of glycogen synthase kinase-3 (GSK3) in mammalian cells is an autophosphorylation event. Biochem J 377:249-255.

Cole AR, Knebel A, Morrice NA, Robertson LA, Irving AJ, Connolly CN, Sutherland C (2004b) GSK-3 phosphorylation of the Alzheimer epitope within collapsin response mediator proteins regulates axon elongation in primary neurons. J Biol Chem 279:50176-50180.

Cole AR, Causeret F, Yadirgi G, Hastie CJ, McLauchlan H, McManus EJ, Hernández F, Eickholt BJ, Nikolic M, Sutherland C (2006) Distinct priming kinases contribute to differential regulation of collapsin response mediator proteins by glycogen synthase kinase-3 in vivo. J Biol Chem 281:16591-16598.

Cyranoski D (2007) Chinese network to start trials of spinal surgery. Nature 446:476-477.

Deo RC, Schmidt EF, Elhabazi A, Togashi H, Burley SK, Strittmatter SM (2004) Structural bases for CRMP function in plexin-dependent semaphorin3A signaling. EMBO J 23:9-22.

Dill J, Wang H, Zhou F, Li S (2008) Inactivation of glycogen synthase kinase 3 promotes axonal growth and recovery in the CNS. J Neurosci 28: $8914-8928$

Dull T, Zufferey R, Kelly M, Mandel RJ, Nguyen M, Trono D, Naldini L (1998) A third-generation lentivirus vector with a conditional packaging system. J Virol 72:8463-8471.

Eickholt BJ, Walsh FS, Doherty P (2002) An inactive pool of GSK-3 at the leading edge of growth cones is implicated in Semaphorin $3 \mathrm{~A}$ signaling. J Cell Biol 157:211-217.

Fournier AE, Takizawa BT, Strittmatter SM (2003) Rho kinase inhibition enhances axonal regeneration in the injured CNS. J Neurosci 23:14161423.

Fukata Y, Itoh TJ, Kimura T, Ménager C, Nishimura T, Shiromizu T, Watanabe H, Inagaki N, Iwamatsu A, Hotani H, Kaibuchi K (2002) CRMP-2 binds to tubulin heterodimers to promote microtubule assembly. Nat Cell Biol 4:583-591.

Gaetano C, Matsuo T, Thiele CJ (1997) Identification and characterization of a retinoic acid-regulated human homologue of the unc-33-like phosphoprotein gene (hUlip) from neuroblastoma cells. J Biol Chem 272: 12195-12201.

Goshima Y, Nakamura F, Strittmatter P, Strittmatter SM (1995) Collapsininduced growth cone collapse mediated by an intracellular protein related to UNC-33. Nature 376:509-514.

GrandPré T, Nakamura F, Vartanian T, Strittmatter SM (2000) Identification of the Nogo inhibitor of axon regeneration as a Reticulon protein. Nature 403:439-444.

Hall C, Brown M, Jacobs T, Ferrari G, Cann N, Teo M, Monfries C, Lim L (2001) Collapsin response mediator protein switches RhoA and Racl morphology in N1E-115 neuroblastoma cells and is regulated by Rho kinase. J Biol Chem 276:43482-43486.

Hedgecock EM, Culotti JG, Thomson JN, Perkins LA (1985) Axonal guidance mutants of Caenorhabditis elegans identified by filling sensory neurons with fluorescein dyes. Dev Biol 111:158-170.

Hsieh SH, Ferraro GB, Fournier AE (2006) Myelin-associated inhibitors regulate cofilin phosphorylation and neuronal inhibition through Lim kinase and Slingshot phosphatase. J Neurosci 26:1006-1015.

Igarashi M, Strittmatter SM, Vartanian T, Fishman MC (1993) Mediation by $\mathrm{G}$ proteins of signals that cause collapse of growth cones. Science 259:77-79.

Inatome R, Tsujimura T, Hitomi T, Mitsui N, Hermann P, Kuroda S, Yamamura H, Yanagi S (2000) Identification of CRAM, a novel unc-33 gene family protein that associates with CRMP3 and protein-tyrosine kinase(s) in the developing rat brain. J Biol Chem 275:27291-27302.
Kim WY, Zhou FQ, Zhou J, Yokota Y, Wang YM, Yoshimura T, Kaibuchi K, Woodgett JR, Anton ES, Snider WD (2006) Essential roles for GSK-3s and GSK-3-primed substrates in neurotrophin-induced and hippocampal axon growth. Neuron 52:981-996.

Kockeritz L, Doble B, Patel S, Woodgett JR (2006) Glycogen synthase kinase-3-an overview of an over-achieving protein kinase. Curr Drug Targets 7:1377-1388.

Leung T, Ng Y, Cheong A, Ng CH, Tan I, Hall C, Lim L (2002) p80 ROKalpha binding protein is a novel splice variant of CRMP-1 which associates with CRMP-2 and modulates RhoA-induced neuronal morphology. FEBS Lett 532:445-449.

Liu BP, Cafferty WB, Budel SO, Strittmatter SM (2006) Extracellular regulators of axonal growth in the adult central nervous system. Philos Trans R Soc Lond B Biol Sci 361:1593-1610.

Mimura F, Yamagishi S, Arimura N, Fujitani M, Kubo T, Kaibuchi K, Yamashita T (2006) Myelin-associated glycoprotein inhibits microtubule assembly by a Rho-kinase-dependent mechanism. J Biol Chem 281: $15970-15979$.

Minturn JE, Fryer HJ, Geschwind DH, Hockfield S (1995) TOAD-64, a gene expressed early in neuronal differentiation in the rat, is related to unc-33, a C. elegans gene involved in axon outgrowth. J Neurosci 15:6757-6766.

Neve RL, Howe JR, Hong S, Kalb RG (1997) Introduction of the glutamate receptor subunit 1 into motor neurons in vitro and in vivo using a recombinant herpes simplex virus. Neuroscience 79:435-447.

Owen R, Gordon-Weeks PR (2003) Inhibition of glycogen synthase kinase 3 beta in sensory neurons in culture alters filopodia dynamics and microtubule distribution in growth cones. Mol Cell Neurosci 23:626-637.

Pan SH, Chao YC, Chen HY, Hung PF, Lin PY, Lin CW, Chang YL, Wu CT, Lee YC, Yang SC, Hong TM, Yang PC (2010) Long form collapsin response mediator protein-1 (LCRMP-1) expression is associated with clinical outcome and lymph node metastasis in non-small cell lung cancer patients. Lung Cancer 67:93-100.

Quinn CC, Gray GE, Hockfield S (1999) A family of proteins implicated in axon guidance and outgrowth. J Neurobiol 41:158-164.

Quinn CC, Chen E, Kinjo TG, Kelly G, Bell AW, Elliott RC, McPherson PS, Hockfield S (2003) TUC-4b, a novel TUC family variant, regulates neurite outgrowth and associates with vesicles in the growth cone. J Neurosci 23:2815-2823.

Rosslenbroich V, Dai L, Baader SL, Noegel AA, Gieselmann V, Kappler J (2005) Collapsin response mediator protein-4 regulates F-actin bundling. Exp Cell Res 310:434-444.

Shi SH, Cheng T, Jan LY, Jan YN (2004) APC and GSK-3beta are involved in $\mathrm{mPar} 3$ targeting to the nascent axon and establishment of neuronal polarity. Curr Biol 14:2025-2032.

Siddiqui SS, Culotti JG (1991) Examination of neurons in wild type and mutants of Caenorhabditis elegans using antibodies to horseradish peroxidase. J Neurogenet 7:193-211.

Sivasankaran R, Pei J, Wang KC, Zhang YP, Shields CB, Xu XM, He Z (2004) PKC mediates inhibitory effects of myelin and chondroitin sulfate proteoglycans on axonal regeneration. Nat Neurosci 7:261-268.

Stenmark P, Ogg D, Flodin S, Flores A, Kotenyova T, Nyman T, Nordlund P, Kursula P (2007) The structure of human collapsin response mediator protein 2, a regulator of axonal growth. J Neurochem 101:906-917

Wang LH, Strittmatter SM (1997) Brain CRMP forms heterotetramers similar to liver dihydropyrimidinase. J Neurochem 69:2261-2269.

Yoshimura T, Kawano Y, Arimura N, Kawabata S, Kikuchi A, Kaibuchi K (2005) GSK-3beta regulates phosphorylation of CRMP-2 and neuronal polarity. Cell 120:137-149.

Yuasa-Kawada J, Suzuki R, Kano F, Ohkawara T, Murata M, Noda M (2003) Axonal morphogenesis controlled by antagonistic roles of two CRMP subtypes in microtubule organization. Eur J Neurosci 17:2329-2343.

Zhou FQ, Snider WD (2005) Cell biology. GSK-3beta and microtubule assembly in axons. Science 308:211-214.

Zhou FQ, Zhou J, Dedhar S, Wu YH, Snider WD (2004) NGF-induced axon growth is mediated by localized inactivation of GSK-3beta and functions of the microtubule plus end binding protein APC. Neuron 42:897-912. 